\title{
Feasibility and Oncological Safety of Axillary Reverse Mapping in Patients With Locally Advanced Breast Cancer and Partial Response After Neoadjuvant Chemotherapy
}

\author{
MARCO PELLICCIARO ${ }^{1}$, MARCO MATERAZZO $^{1}$, CHIARA BUONOMO $^{2}$ and GIANLUCA VANNI ${ }^{1}$ \\ ${ }^{1}$ Breast Unit, Department of Surgical Science, Policlinico Tor Vergata University, Rome, Italy; \\ ${ }^{2}$ Department of Emergency and Admission, Critical Care Medicine, \\ Pain Medicine and Anesthetic Science, Policlinico Tor Vergata University, Rome, Italy
}

\begin{abstract}
Background/Aim: Axillary reverse mapping (ARM) aims to identify and preserve arm drainage in order to prevent lymphedema following axillary lymph node dissection. Oncological-safety and feasibility are still debated, especially in patients with locally-advanced breast cancer $(L A B C)$. We report the first case of the AXMAP 1.0 study performed in our Institution. Patients and Methods: A 52-year-old patient with a triple-negative LABC and partial response to neoadjuvant chemotherapy underwent axillary lymph-node dissection using fluorescence ARM. Results: Two lymph-nodes draining the ipsilateral upper arm were identified and were not preserved due to suspicion of malignancy. Pathological examination confirmed the presence of malignancy in both lymph nodes. Conclusion: Further studies should be designed in order to validate the oncological safety of this technique, especially in patients with $L A B C$ requiring neoadjuvant chemotherapy.
\end{abstract}

Despite advances in treatment, up to $30 \%$ of patients with breast cancer (BC) experience distant disease relapse and axillary lymph node involvement still remains the most powerful predictor of distant relapse (1). Traditional axillary staging consists of Level I and II axillary lymph nodes dissection (AD). AD aims to accurately establish the presence of axillary disease and has been associated with an

This article is freely accessible online.

Correspondence to: Marco Materazzo, Breast Unit, Department of Surgical Science, PTV: Policlinico Tor Vergata University, Viale Oxford 81, 00133 Rome, Italy. Tel: +39 3395685883, e-mail: marco.materazzzo@ptvonline.it

Key Words: Breast neoplasm, axillary reverse mapping, axillary lymph node dissection, local advanced breast cancer, axillary imaging. increased long-term local disease control rate. However, AD is associated with disabling complications (e.g. upper arm lymphedema, seromas and postoperative infections) (2).

Since the last century, several surgical protocols has been designed in breast surgery to reduce postoperative stress (3), minimize surgical extent of the primary tumor (4-7), enhance breast reconstruction $(8,9)$, or even to entirely avoid surgery (10-12). In this period, a faster recovery is even more demanded by patients and healthcare workers due to the perceived risk of coronavirus cross-infection (13-15).

Regarding axillary surgery, in the beginning of the second millennium the sentinel lymph node biopsy (SLNB) technique was developed in Milan and soon became the axillary staging gold standard in early BC (EBC), reducing the need of AD (16). Additionally, since the publication of the Z-0011 study (17), several studies investigated the relationship between primary tumor biological subtypes and axillary involvement in order to safely avoid AD in case of SLNB involvement (18-20). However, despite the fact SLNB has reduced the $\mathrm{AD}$ rate and incidence of postoperative complication, up to $13 \%$ of patients will anyway experience upper arm lymphedema (17), requiring an innovative surgical approach as axillary reverse mapping (ARM) and selective AD (SAD).

ARM was developed for detecting and sparing lymphatic drainage of the upper arm with SAD, reducing the leading cause of upper arm lymphedema following axillary procedure which is the interruption of lymphatic drainage of the arm (21). This emerging concept, if validated, may prevent upper lymphedema in patients undergoing SAD as "arm nodes" should be free from metastasis (22). No published guidelines have promoted the ARM technique in BC so far, thus oncological safety should still be assessed.

Moreover, oncological safety and feasibility of ARM in patients who are likely to undergo AD such as in local advanced breast cancer (LABC) or after neoadjuvant chemotherapy (NAC) are still debated in the literature (23). 
Hence, we report our first case of a patient enrolled in our monocentric study (AXMAP 1.0).

\section{Patients and Methods}

Study design and patient selection. A single institution, single arm (patients undergoing ARM procedure) observational trial was undertaken. The local institutional review board (PTV Policlinico Tor Vergata) approved the study with the code name AXMAP 1.0 and registration number CEI $n^{\circ} 119 / 20$. The primary endpoint of the study was to calculate the false-negative rate of axillary involvement in the ARM procedure (number of patients with fluorescent ex vivo positive lymph node/total number of patients). Secondary aims were calculation of the rate of fluorescent-positive node positivity during ex vivo examination (number of fluorescent ex vivo positive nodes/total number of fluorescent lymph nodes) and collection of early postoperative complications ( $24 \mathrm{~h})$. Due to the preliminary design of the study, power analysis was not performed and the local institutional review board allowed us to enroll 50 patients.

The primary inclusion criteria for the AXMAP 1.0 study were a diagnosis of non-metastatic BC with clinically or radiologically proven positive lymph nodes in the axilla. Other inclusion criteria were age between 18-70 years old and female gender. Patients with a history of homolateral axillary, breast or arm surgery were excluded from the study. Moreover, patients with a history of upper limp burns were excluded as well. Before recruitment, all patients were informed regarding the surgical approach and signed a specific written informed consent for the enrollment in the study.

Preoperative evaluation. Prior to daily scheduled surgeries (at 7:30 a.m.), venous-blood samples were collected via peripheral vein of the antecubital arm. Samples were processed to obtain leukocytes and lymphocyte subsets in the same technique previously published in our manuscript $(3,24)$.

Surgical techniques. All patients were placed in supine decubitus position. Following the induction of anesthesia and immediately prior to the surgery, intradermal injection of $0.1 \mathrm{ml}(0.25 \mathrm{mg})$ of indocyanine green (ICG) (Diagnostic Green GmbH, AschheimDornach, Germany) was applied in the ipsilateral second interdigital space (Figure 1) and the area of injection was massaged while an invisible near-infrared fluorescence imaging system Probe (IC Flow, SEDA SPA, Diagnostic Green GmbH, Aschheim-Dornach, Germany) was used to assess the success of the ARM procedure. The number of fluorescent preoperatory lymph nodes was collected. All operatory room staff involved in the surgical procedure were outside the surgical room during the ARM procedure and were unaware of the ARM results (single blind). Following ARM, patients underwent breast surgery according to the current standard of care. Axillary surgeries were performed by an attending surgeon with more than 15 years of expertise in oncological breast surgery. The limits of $\mathrm{AD}$, as described by Mohamed et al., were axillary vein superiorly, lateral border of subscapularis muscle laterally and medial border of pectoralis minor medially. The long and lateral thoracic nerves were spared and the intercostobrachial nerve was preserved if recognized (25).

Ex vivo evaluation. In order to evaluate the presence of nodes draining the upper arm in the $\mathrm{AD}$ specimen, ex vivo evaluations of fluorescent preoperatory lymph nodes were collected by a physician

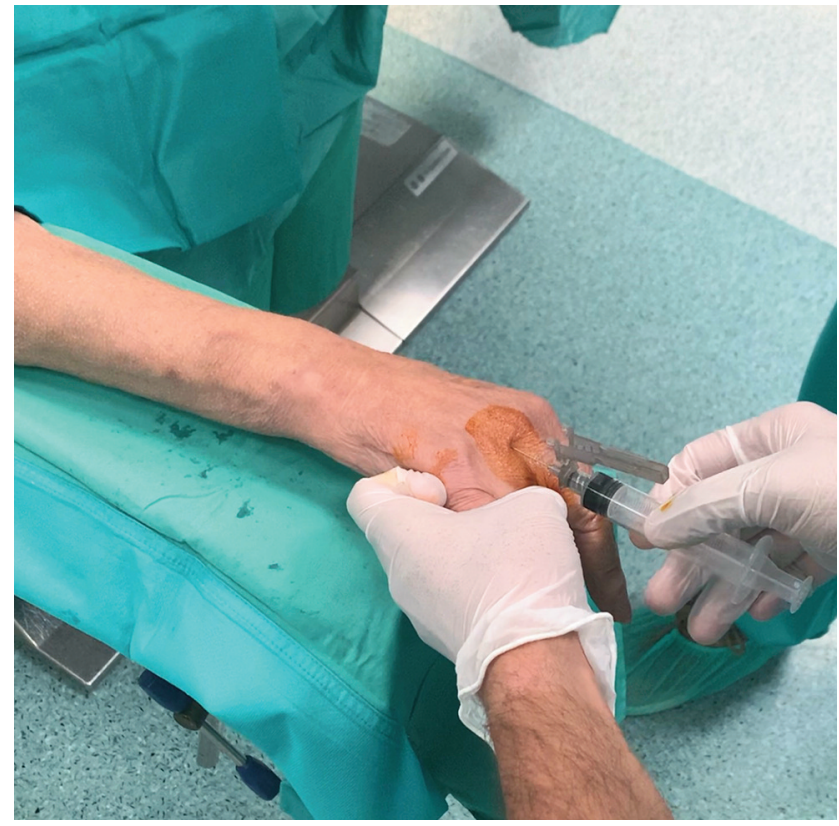

Figure 1. Indocyanine green (ICG) injection (Diagnostic Green $\mathrm{GmbH}$, Aschheim-Dornach, Germany). Intradermal injection of $0.1 \mathrm{ml}(0.25$ $\mathrm{mg}$ ) of ICG in the interdigital second space.

not directly involved in the surgical treatment (Figure 2). Surgical specimens were divided according to the Berg classification level and fluorescent lymph node were sent separately.

Data collection. Demographic and preoperative data were collected. Data from pathological examinations were collected according to the state-of-art classifications (26). All specimens were evaluated separately by two different pathologists with more than 10 years of expertise in breast pathology, which were unaware of the ex vivo evaluation.

One month follow-up visit was performed to assess $>2$ Clavien-Dindo postoperative complications $(27,28)$ and secondary postoperative lymphedema (29).

\section{Results}

Patient characteristics and neoadjuvant chemotherapy. A 52year-old G3P3 post-menopausal woman in good clinical condition was evaluated in our clinic for new-onset palpable breast lesions. Family history was unremarkable regarding oncological disease. Her past medical history reported renal artery aneurysm treated with covered stents (30).

Physical examination revealed skin dimpling and a palpable lesion in the right breast (upper outer quadrant). Mammography exhibited a compact cluster of microcalcification in the upper outer quadrant of the right breast. Ultrasonography performed in the same session revealed a nodule BIRADS-IV with 25 $\mathrm{mm} \times 30 \mathrm{~mm}$ diameter and homolateral malignant-suspicious lymph-nodes [maximum diameter (dtm) $40 \mathrm{~mm}$ ]. 


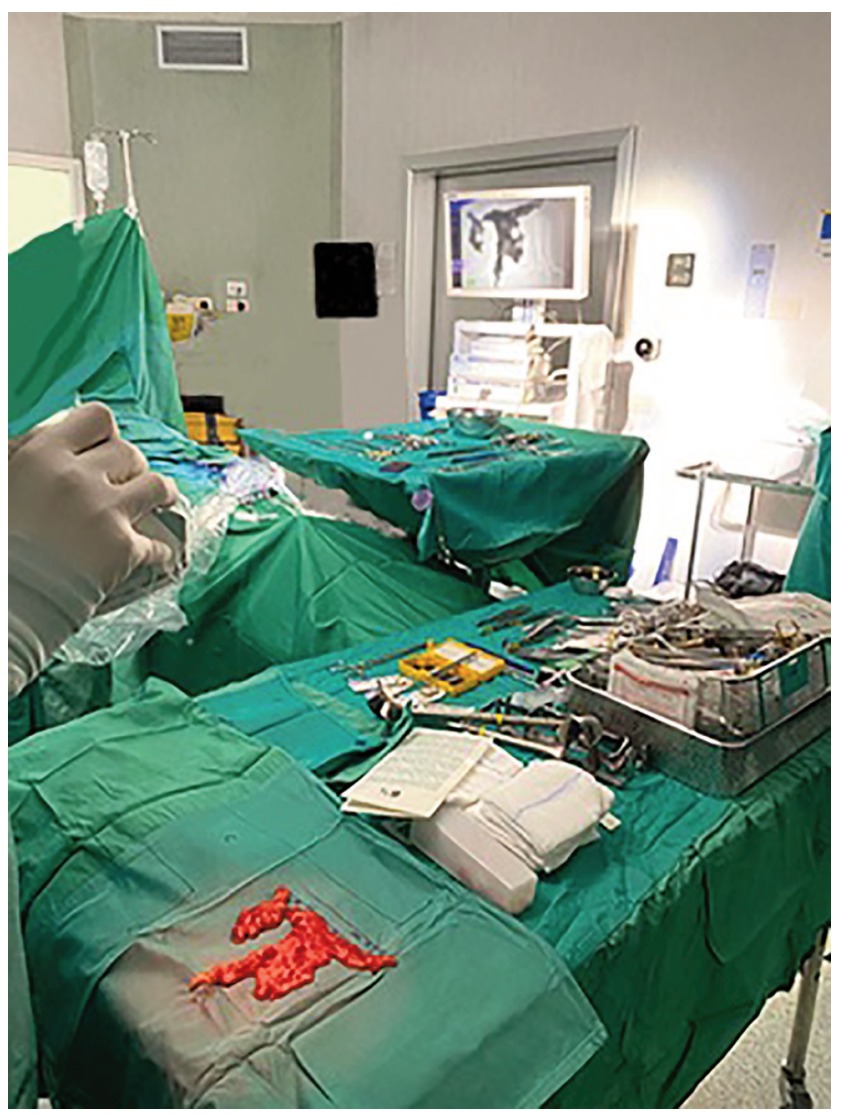

Figure 2. Surgical specimen scanning. Axillary lymph-nodes underwent an ex vivo scanning with an invisible near-infrared fluorescence imaging system (IC Flow, SEDA SPA, Diagnostic Green GmbH, AschheimDornach, Germany).

The patient underwent a biopsy of the lesion and the pathological examination revealed a triple-negative invasive ductal carcinoma [estrogen receptor (ER)-negative, progesterone receptor (PR)-negative, human epidermal growth factor receptor-2 (HER-2) score 0 and Ki67 90\%]. Due to claustrophobia, the patient refused to undergo a magnetic resonance imaging and underwent a positron emission tomography (PET)-computed tomography (CT). PET-CT described a pathological uptake by the breast lesions; ipsilateral axillary, Rotter's and supraclavicular lymph nodes (SUV max: 11.7, 14.1, 12.5, and 6.4, respectively).

The clinical case was discussed during the breast cancer multidisciplinary team (MDT) meeting and neoadjuvant chemotherapy (NAC) was planned. NAC regimen included four cycles of adriamycin and cyclophosphamide (AC) followed by six cycles of carboplatin and paclitaxel (CarboPTX). After five months, neoadjuvant chemotherapy was suspended due to grade 4 myelotoxicity, and right mastectomy, tissue expander placement and ANLD were planned. Preoperative bilateral breast ultrasonography
Table I. Baseline patient features.

\begin{tabular}{lc}
\hline Age (yrs) & $52.4 \mathrm{yrs}$ \\
Weight $(\mathrm{Kg})$ & $73.6 \mathrm{~kg}$ \\
BMI & 26.57 \\
Stage at diagnosis & $\mathrm{cT} 2 \mathrm{cN} 1 \mathrm{cM0}(\mathrm{IIB})$ \\
Stage after neoadjuvant chemotherapy & pT1c pN2a pM0 (IB) \\
Procedure time (min.) & $131 \mathrm{~min}$ \\
Total lymph node & 31 \\
Positive lymph node $(\%)$ & $5(16.13 \%)$ \\
Total fluorescent lymph node $(\%)$ & $2(6.45 \%)$ \\
Positive fluorescent lymph node $(\%)$ & $2(100 \%)$ \\
\hline
\end{tabular}

BMI: Body mass index.

revealed a partial pathological response (lesion diameter 19 $\mathrm{mm} \times 10 \mathrm{~mm}$, lymph node maximum diameter $19 \mathrm{~mm}$ ).

Aiming at the reduction of lymphedema following AD, after discussion of potential benefit and risk of the procedure and signature of informed consent, the patient was enrolled in the AXMAP 1.0 study. Baseline features are enlisted in Table I.

Preoperative evaluation and surgical procedure. Preoperative blood work was unremarkable regarding leukocyte and lymphocyte subsets. Following the induction of anesthesia and immediately prior to the surgery, intradermal injection of $0.1 \mathrm{ml}(0.25 \mathrm{mg})$ of ICG identified two different lymph-nodes draining the ipsilateral upper arm. According to preoperative assessment the patient underwent AD.

Ex vivo evaluation. Following the procedure, ex vivo scanning with invisible near-infrared fluorescence imaging system was performed (Figure 2). The imaging system confirmed the presence of fluorescence: one at level I and one at level II (Figure 3). Fluorescent lymph nodes were $e x$ vivo divided and analyzed separately by the pathologist. The surgical program continued with right simplex mastectomy followed by placement of a tissue expander.

Histological evaluation. Histological examination of the primary tumor showed a $17-\mathrm{mm}$ invasive ductal carcinoma grade 3 (G3), (tubules: score 3, pleomorphism: score 3; mitosis score 3. Immunohistochemical staining confirmed triple negative status of the neoplasia (ER-negative, PR-negative, cErb-B2 score 0 , confirmed independently by two expert pathologists) and Ki67 of approximately $87 \%$. A total of 5 out of 31 lymph nodes were sites of metastasis encompassing 2 non-fluorescent pathological nodes at level I, 1 non-fluorescent pathological node at level II and both fluorescent nodes separated during ex vivo procedure (ypT1cN2a).

Postoperative follow-up. The patient was discharged during postoperative day II in a good clinical condition without 


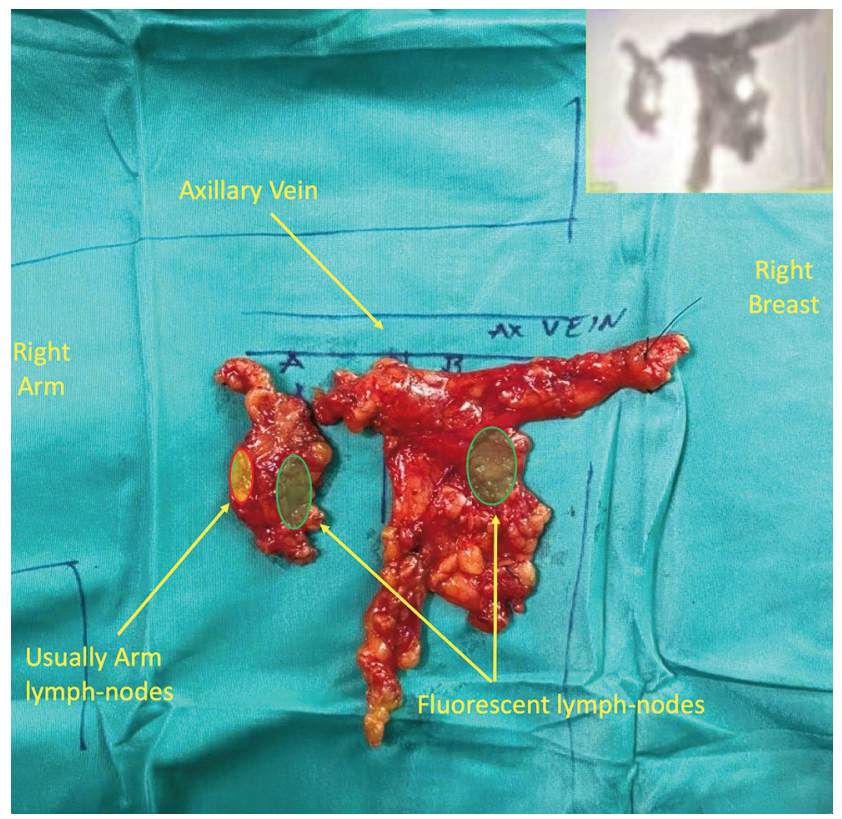

Figure 3. Surgical specimen analysis. Axillary lymph-nodes underwent an ex vivo scanning with invisible near-infrared fluorescence imaging system (IC Flow, SEDA SPA, Diagnostic Green GmbH, AschheimDornach, Germany), upper quadrant fluorescence of the specimen.

reported complications. At 1-month follow-up, the patient was free from distant and loco-regional neoplastic recurrence, with normal function of the right arm and stage 1 lymphedema.

\section{Discussion}

Since the beginning of the second millennium, development of SLNB procedure in EBC and Z011 trial evidence determined a steady decrease of AD in the USA ( $2.43 \%$ yearly reduction) (31), and thus a reduction in axillary complications $(2,32)$.

Common AD complications consist of superior arm lymphedema, shoulder dysfunction, paresthesia and discomfort $(2,19)$. In particular, superior arm lymphedema due to lesion of nodes draining the arm is the most common (complication rate 2\%-56\%) and strongly affects quality of life of patients $(32,33)$.

In order to reduce lymphedema rate in patients undergoing AD, non-invasive axillary assessment with PET-TC (34) and ARM techniques and SAD were developed (22). ARM identifies axillary arm lymphatic drainage with a low likelihood of receiving lymph from the breast (22), that may be spared during $\operatorname{SAD}(35,36)$. With this promising assumption, Gennaro et al. reported in 2013 a two-thirds reduction in upper arm lymphedema following the ARMSAD technique (36). Two main theories could explain this incomplete result: incomplete identification of arm nodes or a cross-over between arm and breast drainage systems (23).
Although Gennaro et al. reported in their monocentric study in 2020 a $1.36 \%$ of axillary failure after ARM-SAD procedure in 100 consecutive positive nodes in patients after long term follow up (35), oncological safety and feasibility of ARM are not fully understood. Currently no published guidelines endorse ARM in BC patients and its exact role is still debated, especially in patients which are likely to undergo $A D$ as $\mathrm{LABC}$ or after $\operatorname{NAC}(21,23)$. The main limitations of ARM techniques include potential understaging of the disease and potential residual disease in the axilla due to the cross-over between arm and breast lymphatic systems (23). In fact, both extensive axillary disease and primary medical treatment could influence the formation of neoplastic emboli, debris depositions and fibrosis or shrinkage of lymphatic routes (37).

Our patient presented with a LABC with a partial radiological response after NAC, and the attending surgeon, which was blindly performing the procedure, did not spare the fluorescent lymph node due to anatomical and oncological reasons. Regarding anatomy, our case involved lymph nodes of level I and II according to Berg classification (Figure 3), contrarily to the preoperational expectations. In fact, a recent anatomical classification postulates two separate lymphatic drainage systems in the axilla with a medial portion receiving from the breast, around the lateral thoracic vein, and spreading upwards and medially behind the pectoralis minor muscle to level II nodes (zones A and B), and a lateral part (zones C and D) receiving lymph from the upper arm (38). Moreover, lack of clinical response in the axilla could probably impact the lymphatic drainage with a potential cross-link between arm and breast lymphatic drainage systems, causing this anatomical variation. Even in case of complete pathological response, it is uncertain whether the oncological remodeling of the lymph drainage system in the axilla regresses post neoadjuvant therapy $(21,23)$. Indeed, in a recent systematic review, ARM node metastases rate was similar with or without NAC (39). In view of this evidence, oncological safety of ARM technique in our opinion should be considered firstly in patients with a lower risk of progression, as in patients with a complete radiological response post NAC (40).

Moreover, in our case, both fluorescent nodes presented physical malignancy characteristics, thus they should be removed regardless of their anatomical position in the axilla $(1,2)$. The majority of case series reported in the literature evaluated patients without clinical axillary lymph nodes involvement $(21,23)$. In patients with LABC, the lack of reported data prevents affirmation of the oncological safety of the procedure. Further studies should be designed, aiming to validate the oncological safety of this technique or to analyze the characteristics of patients who could benefit from arm nodes preservation. 


\section{Conflicts of Interest}

The Authors declare no conflicts of interest regarding this study.

\section{Authors' Contributions}

Study conception and design: Vanni Gianluca; Collection of data: Pellicciaro Marco; Analysis of data: Vanni Gianluca, Materazzo Marco, Pellicciaro Marco Interpretation of data: Materazzo Marco, Pellicciaro Marco, Gianluca Vanni; Critical revision: Vanni Gianluca; Critical revision of literature: Buonomo Chiara, Materazzo Marco.

\section{Acknowledgements}

None declared.

\section{References}

1 Buonomo O, Caredda E, Portarena I, Vanni G, Orlandi A, Bagni C, Petrella G, Palombi L and Orsaria P: New insights into the metastatic behavior after breast cancer surgery, according to well-established clinicopathological variables and molecular subtypes. PLOS ONE 12(9): e0184680, 2019. DOI: 10.1371/ journal.pone. 0184680

2 Orsaria P, Varvaras D, Vanni G, Pagnani G, Scaggiante J, Frusone F, Granai A, Petrella G and Buonomo O: Nodal status assessment in breast cancer: Strategies of clinical grounds and quality of life implications. International Journal of Breast Cancer 2014: 1-8, 2017. DOI: 10.1155/2014/469803

3 Vanni G, Materazzo M, Perretta T, Meucci R, Anemona L, Buonomo C, Dauri M, Granai AV, Rho M, Ingallinella S, Tacconi F, Ambrogi V, Chiaravalloti A, Schillaci O, Petrella G and Buonomo OC: Impact of awake breast cancer surgery on postoperative lymphocyte responses. In Vivo 33(6): 1879-1884, 2019. PMID: 31662515. DOI: 10.21873/invivo.11681

4 Roselli M, Guadagni F, Buonomo O, Belardi A, Ferroni P, Diodati A, Anselmi D, Cipriani C, Casciani CU, Greiner J and Schlom J: Tumor markers as targets for selective diagnostic and therapeutic procedures. Anticancer Res 16(4B): 2187-2192, 1996. PMID: 8694541.

5 Buonomo O, Granai AV, Felici A, Piccirillo R, De Liguori Carino N, Guadagni F, Polzoni M, Mariotti S, Cipriani C, Simonetti G, Cossu E, Schiaroli S, Altomare V, Cabassi A, Pernazza E, Casciani CU and Roselli M: Day-surgical management of ductal carcinoma in situ (DCIS) of the breast using wide local excision with sentinel node biopsy. Tumori 88(3): S48-S49, 2002. PMID: 12365390.

6 Orsaria P, Chiaravalloti A, Fiorentini A, Pistolese C, Vanni G, Granai AV, Varvaras D, Danieli R, Schillaci O, Petrella G and Buonomo OC: PET probe-guided surgery in patients with breast cancer: proposal for a methodological approach. In Vivo 31(1): 101-110, 2017. PMID: 28064227. DOI: 10.21873/invivo.11031

7 Buonomo O, Cabassi A, Guadagni F, Piazza A, Felici A, Piccirillo R, Atzei GP, Cipriani C, Schiaroli S, Mariotti S, Guazzaroni MN, Cossu E, Simonetti G, Pernazza E, Casciani $\mathrm{CU}$ and Roselli M: Radioguided-surgery of early breast lesions. Anticancer Res 21(3C): 2091-2097, 2001. PMID: 11501831.

8 Buonomo OC, Varvaras D, Montuori M, Vanni G, Venditti D, Elia S, Santurro L, Granai A V, Petrella G and Rossi P: One- stage immediate implant-based breast reconstruction, using biological matrices after conservative mastectomies: Preliminary experience of the University Hospital of Tor Vergata, Rome. Minerva Chir 28(6): 221-226, 2015.

9 Bielli A, Bernardini R, Varvaras D, Rossi P, Di Blasi G, Petrella G, Buonomo OC, Mattei M and Orlandi A: Characterization of a new decellularized bovine pericardial biological mesh: Structural and mechanical properties. J Mech Behav Biomed Mater 78: 420-426, 2018. PMID: 29223730. DOI: 10.1016/ j.jmbbm.2017.12.003

10 Perretta T, Lamacchia F, Ferrari D, Beninati E, DI Tosto F, DE Stasio V, Meucci R, DI Stefano C, Buonomo OC, Vanni G and Pistolese CA: Evaluation of ultrasound-guided 8-Gauge Vacuum-assisted excision system for the removal of USdetectable breast lesions. Anticancer Res 40(3): 1719-1729, 2020. PMID: 32132080. DOI: 10.21873/anticanres.14125

11 Perretta T, Meucci R, Pistolese CA, Manenti G, Stefano CD, Vanni G, Anemona L, Ferrari D, Lamacchia F, De Stasio V and Buonomo OC: Ultrasound-guided laser ablation after excisional vacuum-assisted breast biopsy for small malignant breast lesions: preliminary results. Technol Cancer Res Treat 20: 1533033820980089, 2021. PMID: 33618620. DOI: 10.1177/ 1533033820980089

12 Quaranta V, Manenti G, Bolacchi F, Cossu E, Pistolese CA, Buonomo OC, Carotenuto L, Piconi C and Simonetti G: FEM analysis of RF breast ablation: multiprobe versus cool-tip electrode. Anticancer Res 27(2): 775-784, 2007. PMID: 17465202.

13 Vanni G, Materazzo M, Pellicciaro M, Ingallinella S, Rho M, Santori F, Cotesta M, Caspi J, Makarova A, Pistolese CA and Buonomo OC: Breast cancer and COVID-19: The effect of fear on patients' decision-making process. In Vivo 34(3 Suppl): 16511659, 2020. PMID: 32503825. DOI: 10.21873/invivo.11957

14 Vanni G, Pellicciaro M, Materazzo M, Palombi L and Buonomo OC: Breast cancer diagnosis in Coronavirus-era: Alert from Italy. Front Oncol 10: 938, 2020. PMID: 32574281. DOI: 10.3389/ fonc. 2020.00938

15 Vanni G, Materazzo M, Santori F, Pellicciaro M, Costesta M, Orsaria P, Cattadori F, Pistolese CA, Perretta T, Chiocchi M, Meucci R, Lamacchia F, Assogna M, Caspi J, Granai AV, DE Majo A, Chiaravalloti A, D’Angelillo MR, Barbarino R, Ingallinella S, Morando L, Dalli S, Portarena I, Altomare V, Tazzioli $G$ and Buonomo OC: The effect of Coronavirus (COVID-19) on breast cancer teamwork: A multicentric survey. In Vivo 34(3 Suppl): 1685-1694, 2020. PMID: 32503830. DOI: 10.21873/invivo.11962

16 Veronesi U, Viale G, Paganelli G, Zurrida S, Luini A, Galimberti V, Veronesi P, Intra M, Maisonneuve P, Zucca F, Gatti G, Mazzarol G, De Cicco C and Vezzoli D: Sentinel lymph node biopsy in breast cancer: ten-year results of a randomized controlled study. Ann Surg 251(4): 595-600, 2010. PMID: 20195151. DOI: 10.1097/SLA.0b013e3181c0e92a

17 Lucci A, McCall LM, Beitsch PD, Whitworth PW, Reintgen DS, Blumencranz PW, Leitch AM, Saha S, Hunt KK, Giuliano AE and American College of Surgeons Oncology Group: Surgical complications associated with sentinel lymph node dissection (SLND) plus axillary lymph node dissection compared with SLND alone in the American College of Surgeons Oncology Group Trial Z0011. J Clin Oncol 25(24): 3657-3663, 2007. PMID: 17485711. DOI: 10.1200/JCO.2006.07.4062 
18 Van Zee KJ, Manasseh DM, Bevilacqua JL, Boolbol SK, Fey JV, Tan LK, Borgen PI, Cody HS 3rd and Kattan MW: A nomogram for predicting the likelihood of additional nodal metastases in breast cancer patients with a positive sentinel node biopsy. Ann Surg Oncol 10(10): 1140-1151, 2003. PMID: 14654469. DOI: 10.1245/aso.2003.03.015

19 Orsaria P, Caredda E, Genova F, Materazzo M, Capuano I, Vanni G, Granai AV, DE Majo A, Portarena I, Sileri P, Petrella G, Palombi L and Buonomo OC: Additional nodal disease prediction in breast cancer with sentinel lymph node metastasis based on clinicopathological features. Anticancer Res 38(4): 2109-2117, 2018. PMID: 29599329. DOI: 10.21873/anticanres.12451

20 Ielpo B, Pernaute AS, Elia S, Buonomo OC, Valladares LD, Aguirre EP, Petrella G and Garcia AT: Impact of number and site of lymph node invasion on survival of adenocarcinoma of esophagogastric junction. Interact Cardiovasc Thorac Surg 10(5): 704-708, 2010. PMID: 20154347. DOI: 10.1510/icvts. 2009.222778

21 Shao X, Sun B and Shen Y: Axillary reverse mapping (ARM): where to go. Breast Cancer 26(1): 1-10, 2019. PMID: 29961238. DOI: $10.1007 / \mathrm{s} 12282-018-0886-0$

22 Thompson M, Korourian S, Henry-Tillman R, Adkins L, Mumford S, Westbrook KC and Klimberg VS: Axillary reverse mapping (ARM): a new concept to identify and enhance lymphatic preservation. Ann Surg Oncol 14(6): 1890-1895, 2007. PMID: 17479341. DOI: 10.1245/s10434-007-9412-x

23 Gobardhan PD, Wijsman JH, van Dalen T, Klompenhouwer EG, van der Schelling GP, Los J, Voogd AC and Luiten EJ: ARM: axillary reverse mapping - the need for selection of patients. Eur J Surg Oncol 38(8): 657-661, 2012. PMID: 22607749. DOI: 10.1016/j.ejso.2012.04.012

24 Piazza A, Adorno D, Poggi E, Borrelli L, Buonomo O, Pisani F, Valeri M, Torlone N, Camplone C, Monaco PI, Fraboni D and Casciani CU: Flow cytometry crossmatch: a sensitive technique for assessment of acute rejection in renal transplantation. Transplant Proc 30(5): 1769-1771, 1998. PMID: 9723274. DOI: 10.1016/s0041-1345(98)00423-0

25 Abdelhamid MI, Bari AA, Farid MI and Nour H: Evaluation of axillary reverse mapping (ARM) in clinically axillary node negative breast cancer patients - Randomised controlled trial. Int J Surg 75: 174-178, 2020. PMID: 32059974. DOI: 10.1016/ j.ijsu.2020.01.152

26 Vanni G, Materazzo M, Pellicciaro M, Morando L, Portarena I, Anemona L, D'Angelillo MR, Barbarino R, Chiaravalloti A, Meucci R, Perretta T, Deiana C, Orsaria P, Caspi J, Pistolese CA and Buonomo OC: Does age matter? Estimating risks of locoregional recurrence after breast-conservative surgery. In Vivo 34(3): 1125-1132, 2020. PMID: 32354901. DOI: 10.21873/ invivo.11884

27 Winter R, Haug I, Lebo P, Grohmann M, Reischies FMJ, Cambiaso-Daniel J, Tuca A, Rienmüller T, Friedl H, Spendel S, Forbes AA, Wurzer P and Kamolz LP: Standardizing the complication rate after breast reduction using the Clavien-Dindo classification. Surgery 161(5): 1430-1435, 2017. PMID: 28043695. DOI: 10.1016/j.surg.2016.11.028

28 Panhofer P, Ferenc V, Schütz M, Gleiss A, Dubsky P, Jakesz R, Gnant $\mathrm{M}$ and Fitzal F: Standardization of morbidity assessment in breast cancer surgery using the Clavien Dindo Classification. Int J Surg 12(4): 334-339, 2014. PMID: 24486930. DOI: $10.1016 / j . i j s u .2014 .01 .012$
29 Greene AK and Goss JA: Diagnosis and staging of lymphedema. Semin Plast Surg 32(1): 12-16, 2018. PMID: 29636648. DOI: 10.1055/s-0038-1635117

30 Gandini R, Morosetti D, Chiocchi M, Chiaravalloti A, Citraro D, Loreni G, DA Ros V, Salvatori E and Simonetti G: Long-term follow-up of endovascular treatment of renal artery aneurysms with covered stent deployment. J Cardiovasc Surg (Torino) 57(5): 625-633, 2016. PMID: 25475915.

31 Nocera NF, Pyfer BJ, De La Cruz LM, Chatterjee A, Thiruchelvam PT and Fisher CS: NSQIP analysis of axillary lymph node dissection rates for breast cancer: implications for resident and fellow participation. J Surg Educ 75(5): 1281-1286, 2018. PMID: 29605705. DOI: 10.1016/j.jsurg.2018.02.020

32 Sakorafas GH, Peros G and Cataliotti L: Sequelae following axillary lymph node dissection for breast cancer. Expert Rev Anticancer Ther 6(11): 1629-1638, 2006. PMID: 17134366. DOI: $10.1586 / 14737140.6 .11 .1629$

33 McLaughlin SA, Wright MJ, Morris KT, Giron GL, Sampson MR, Brockway JP, Hurley KE, Riedel ER and Van Zee KJ: Prevalence of lymphedema in women with breast cancer 5 years after sentinel lymph node biopsy or axillary dissection: objective measurements. J Clin Oncol 26(32): 5213-5219, 2008. PMID: 18838709. DOI: $10.1200 / J C O .2008 .16 .3725$

34 Orsaria P, Chiaravalloti A, Caredda E, Marchese PV, Titka B, Anemona L, Portarena I, Schillaci O, Petrella G, Palombi L and Buonomo OC: Evaluation of the usefulness of FDG-PET/CT for nodal staging of breast cancer. Anticancer Res 38(12): 66396652, 2018. PMID: 30504372. DOI: 10.21873/anticanres.13031

35 Gennaro M, Listorti C, Mariani L, Maccauro M, Bianchi G, Capri G, Maugeri I, Lozza L, De Santis MC and Folli S: Oncological safety of selective axillary dissection after axillary reverse mapping in node-positive breast cancer. Eur J Surg Oncol, 2020. PMID: 33160781. DOI: 10.1016/j.ejso.2020.10.031

36 Gennaro M, Maccauro M, Sigari C, Casalini P, Bedodi L, Conti $\mathrm{AR}$, Caraceni A and Bombardieri E: Selective axillary dissection after axillary reverse mapping to prevent breast-cancer-related lymphoedema. Eur J Surg Oncol 39(12): 1341-1345, 2013. PMID: 24113621. DOI: 10.1016/j.ejso.2013.09.022

37 Shirzadi A, Mahmoodzadeh H and Qorbani M: Assessment of sentinel lymph node biopsy after neoadjuvant chemotherapy for breast cancer in two subgroups: Initially node negative and node positive converted to node negative - A systemic review and meta-analysis. J Res Med Sci 24: 18, 2019. PMID: 30988686. DOI: $10.4103 /$ jrms.JRMS_127_18

38 Clough KB, Nasr R, Nos C, Vieira M, Inguenault C and Poulet B: New anatomical classification of the axilla with implications for sentinel node biopsy. Br J Surg 97(11): 1659-1665, 2010. PMID: 20799288. DOI: 10.1002/bjs.7217

39 Wijaya WA, Peng J, He Y, Chen J and Cen Y: Clinical application of axillary reverse mapping in patients with breast cancer: A systematic review and meta-analysis. Breast 53: 189200, 2020. PMID: 32858404. DOI: 10.1016/j.breast.2020.08.007

40 Hayashi M, Yamamoto Y and Iwase H: Clinical imaging for the prediction of neoadjuvant chemotherapy response in breast cancer. Chin Clin Oncol 9(3): 31, 2020. PMID: 32594748. DOI: $10.21037 / \mathrm{cco}-20-15$

Received April 12, 2021

Revised March 3, 2021

Accepted April 18, 2021 